\title{
Práctica preprofesional en la Universidad Nacional de Educación (UNAE): análisis y reflexiones de la modalidad virtual en tiempos de pandemia
}

Pre-professional Practice at UNAE: analysis and reflections on the virtual modality in times of pandemic

\section{Volumen 22, Número 1}

Enero - Abril

pp. 1-27

\author{
María Dolores Pesántez Palacios \\ Paola Alexandra Cuenca Alvarado
}

\section{Citar este documento según modelo APA}

Pesántez Palacios, María Dolores., y Cuenca Alvarado, Paola Alexandra. (2022). Práctica preoprofesional en la Universidad Nacional de Educación (UNAE): análisis y reflexiones de la modalidad virtual en tiempos de pandemia. Revista Actualidades Investigativas en Educación, 22(1), 1-27. Doi. https://doi.org/10.15517/aie.v22i1.47438 


\title{
Práctica preprofesional en la Universidad Nacional de Educación (UNAE): análisis y reflexiones de la modalidad virtual en tiempos de pandemia \\ Pre-professional Practice at UNAE: analysis and reflections on the virtual modality in times of pandemic
}

\author{
María Dolores Pesántez Palacios ${ }^{1}$ \\ Paola Alexandra Cuenca Alvarado²
}

\begin{abstract}
Resumen: Este artículo aporta elementos de una investigación realizada en el año 2020 en la que se amplía el debate sobre la importancia de la práctica preprofesional (PP) o práctica pedagógica en modalidad presencial para la formación de docentes con perfil inclusivo. En la Universidad Nacional de Educación (UNAE) de Ecuador, las PP son consideradas el eje vertebrador de la formación profesional. En el año 2020, debido a la pandemia, la carrera de Educación Especial implementó, en el periodo académico abril-agosto 2020, la modalidad virtual para las PP con el apoyo de las unidades educativas regulares y de educación especializada. En este estudio, de corte cualitativo, se aplica el método participativo desde las perspectivas de estudiantes, tutores y tutoras profesionales (docentes de las escuelas) y tutoras y tutores académicos (docentes de la UNAE). La información se obtuvo de diversas fuentes: cuestionarios en línea, revisión documental y un grupo de discusión, participaron un total de 546 personas. Los resultados sugieren que la práctica preprofesional en modalidad virtual no garantiza los procesos de formación de calidad para los futuros docentes. Las PP en modalidad presencial propician de manera más eficiente espacios de reflexión sobre las experiencias relacionadas con la práctica y la relación que se establece entre teoría y práctica como pilares fundamentales en la formación de docentes. En cambio, las PP en modalidad virtual limitan el contacto del practicante con la realidad socioeducativa, situación que ha impedido establecer procesos adecuados de reflexión sobre la práctica docente e identificación de las necesidades y problemáticas en los diferentes contextos socioeducativos.
\end{abstract}

Palabras clave: educación inclusiva, educación especial, formación de docentes, práctica pedagógica

\begin{abstract}
This article provides elements of a research conducted in 2020 in which the debate on the importance of pre-professional practice (PP) or pedagogical practice in face-to-face mode for the training of research teachers with an inclusive profile is expanded. At the National University of Education (UNAE-Ecuador), PP is considered the backbone of professional training. In the year 2020 due to the pandemic, the Special Education career implemented in the academic period April-August 2020 the virtual modality for PP, with the support of the Regular Educational Units and Specialized Education. This is a qualitative study, applying the participatory method from the perspectives of the main actors of the PP: students, professional tutors (school teachers) and academic tutors (UNAE teachers). Information was obtained from various sources: online questionnaires, documentary review and a focus group. The results suggest that pre-professional practice in virtual mode does not guarantee quality training processes for future teachers. Face-to-face PP provides more efficient spaces for reflection on experiences related to practice and the relationship established between theory and practice as fundamental pillars in teacher training. On the other hand, virtual PP limits the contact of the practitioner with the socio-educational reality, a situation that has prevented the establishment of adequate processes of reflection on teaching practice and identification of needs and problems in the different socio-educational contexts.
\end{abstract}

Key words: inclusive education, special education, teacher training, pedagogical practice

\footnotetext{
1 Docente e investigadora de la Universidad Nacional de Educación, Chuquipata Centro, Ecuador. PhD en Equidad e Innovación Educativa en la Universidad de Vigo, España. Dirección electrónica:maria.pesantez@unae.edu.ec ORCID: https://orcid.org/0000-0001-5914-0663

${ }^{2}$ Investigadora independiente. Actualmente cursa su doctorado en Educación, en la Universidad de Girona, Cataluña, España. Dirección electrónica: paola 88ca@hotmail.com ORCID: https://orcid.org/0000-0002-7320-689X
}

Artículo recibido: 16 de junio, 2021

Enviado a corrección: 15 de setiembre, 2021

Anrobado: 25 de octubre. 2021

Los contenidos de este artículo están bajo una licencia Creative Commons 


\section{Introducción}

La práctica preprofesional en la carrera de Educación Especial se desarrolla bajo la modalidad de una práctica preprofesional diversificada (PPD). Esto significa que, a lo largo de formación profesional, el alumnado transita por diversos contextos socioeducativos, desde las escuelas regulares (de primero a cuarto ciclo), correspondientes a la Unidad de Formación Básica, hasta las escuelas de educación especializada (de quinto a noveno ciclo), correspondientes a la Unidad de Formación Profesional y Unidad de Titulación ${ }^{3}$. Estas experiencias educativas permiten al estudiantado desarrollar el pensamiento práctico a través de la reflexión, el análisis y el cuestionamiento de la realidad en el marco de la inclusión y la atención a la diversidad en el contexto educativo ecuatoriano.

Debido al estado de excepción decretado en el Ecuador a causa de la pandemia del Covid-19, la UNAE y su carrera de Educación Especial implementaron un plan de contingencia para responder el currículo de formación docente en la modalidad virtual. En dicho plan se asumió el reto de organizar las prácticas preprofesionales en ambientes virtuales, con la finalidad de acercar al estudiantado al contexto escuela -el primer y más importante generador de espacios de reflexión sobre la situación educativa que atraviesa nuestro país- y promover su participación en encuentros sincrónicos que les permita el acercamiento al interior de las clases en las instituciones educativas con las que la UNAE trabaja a partir del convenio firmado con el Ministerio de Educación (MinEduc).

Así, el colectivo de docentes de la carrera de Educación Especial, para el periodo académico IS-2020, desarrolló una propuesta de práctica preprofesional combinada (PPC); es decir, una práctica organizada en dos escenarios de aprendizaje:

a. El contexto UNAE: donde se desarrollaron actividades entre estudiantes, tutoras y tutores académicos y expertos en diferentes áreas, a través de plataformas como el Entorno Virtual de Enseñanza y Aprendizaje (EVEA) y el repositorio de la biblioteca UNAE. Las actividades realizadas en el contexto UNAE giraron alrededor del análisis de casos sobre las intervenciones realizadas con las familias y con el alumnado con necesidades educativas especiales (NEE).

b. Las instituciones educativas: donde las actividades se coordinaron entre tutoras y tutores académicos, tutores y tutoras profesionales, y estudiantes, con la finalidad de que todos se involucren en la dinámica de las escuelas a través de encuentros sincrónicos,

\footnotetext{
${ }^{3}$ La carrera de Educación Especial ofrece a partir del séptimo ciclo itinerarios de formación en: Discapacidad Intelectual, Discapacidad Sensorial y Logopedia.
} 
elaboración de material didáctico, apoyo en las clases, revisión de documentos institucionales, curriculares y de política pública; diseño de propuestas de prevención, intervención y atención a la diversidad; y apoyo a las familias para el trabajo con menores.

Este proceso de organización de las prácticas preprofesionales estuvo a cargo de docentes de la UNAE (tutoras y tutores académicos), quienes se encargaron de establecer contacto con las instituciones educativas para socializar el plan de práctica y, posteriormente, monitorear el cumplimiento de dichas actividades. Además, se contó con el apoyo de docentes de Cátedra Integradora e Investigación para los procesos de reflexión de la práctica preprofesional, recolección de información y elaboración de los Proyectos Integradores de Saberes (PIENSA), que emergen de la práctica con base en la identificación de necesidades, problemáticas o situaciones encontradas en los ambientes escolares.

El Modelo de Prácticas Preprofesionales de la UNAE plantea que los Proyectos Integradores de Saberes (PIENSA) "visibilizan y comunican los procesos, metodologías y resultados de las experiencias y reflexiones vividas en la PP, como una experiencia investigativa fundamental para fomentar el pensamiento educativo práctico" (Portilla et al., 2017, p. 20).

Esta investigación muestra los resultados de la evaluación del proceso de prácticas preprofesionales en modalidad virtual implementado en la carrera de Educación Especial, a partir de la información recabada por los actores clave de la práctica (estudiantes, tutores y tutoras), quienes consideraron tres momentos: coordinación, acompañamiento y resultados. Asimismo, se revisaron los informes finales de prácticas elaborados por tutoras y tutores, y se desarrolló un grupo de discusión con el colectivo docente.

Este estudio tiene como finalidad evaluar si la práctica preprofesional en modalidad virtual aporta de manera efectiva al proceso de aprendizaje del alumnado de la carrera de Educación Especial.

\section{Referente teórico: la práctica en la formación profesional}

En este apartado se fundamenta la importancia de los procesos de prácticas preprofesionales. Terranova et al. (2019) aseveran que las prácticas preprofesionales, además de permitir el acercamiento estudiantil a su futuro escenario o contexto laboral, aportan la posibilidad de poner en acción el saber, el saber hacer y el saber actuar que cada estudiante 
construye en su proceso de formación. De tal manera, se considera a la práctica como un proceso complejo que demanda una organización consensuada en la que el estudiantado puede obtener grandes ventajas:

(a) La aproximación a la cultura de la profesión para la que se forman y a las instituciones donde esa profesión se ejerce; (b) lo que han visto y experimentado durante las prácticas les ayudará a generar marcos de referencia cognitivos (decodificadores) que les permitirá entender mejor lo que aprenden en las clases y seminarios de la Universidad; (c) les ofrece experiencias formativas nuevas y complementarias a las académicas; es decir, les permite trabajar objetivos de formación que no se pueden obtener en las clases; (d) les ofrece posibilidades para el autoconocimiento; (e) les ofrece mejores posibilidades de empleo y autoempleo (el hecho de ver cómo funciona una empresa o un servicio puede animarlos a diseñar sus propias iniciativas). (Zabalza, 2017, pp. 22-23)

Por su parte, Latorre (2006) resalta la trascendencia de la práctica al mencionar que es una materia troncal, y considera que este es un espacio ideal donde los futuros docentes pueden tomar contacto con el mundo laboral y desempeñar actividades en contextos reales.

Sin duda, la práctica pedagógica o preprofesional es un componente crucial en la formación de docentes y podría definir sus acciones en el proceso de enseñanza-aprendizaje; tanto así que Pérez (2008) manifiesta que, en su gran mayoría, el desarrollo de competencias son adquiridas por los docentes durante la práctica.

En definitiva, la oportunidad de aproximación al contexto profesional que permiten las prácticas preprofesionales atesora al estudiante un conglomerado de nuevas experiencias por la diversidad de escenarios a los que se acerca, los mismos que amplían el espectro de agentes formativos y enriquecen las competencias y significados de las cosas que van aprendiendo. (Zabalza, 2017, pp. 18-19)

Este recorrido bibliográfico hace énfasis en la relevancia de las prácticas preprofesionales en el currículo de los programas de formación y en el debate de la academia; un proceso con una organización amplia y sistemática que permite que se obtengan importantes espacios de reflexión y aprendizaje para cada practicante. 


\subsection{Reflexiones sobre las prácticas preprofesionales en la formación del docente inclusivo}

El Modelo Pedagógico de la UNAE propone un método innovador en la formación de docentes-investigadores, que considera vivencias y horizontes desde pedagogías inclusivas, críticas, emancipadoras y transformadoras. Esto significa asumir nuevos retos y desafíos para dar respuesta a las demandas de la sociedad en los tiempos actuales.

El perfil de salida de los docentes investigadores de la UNAE está basado en el desarrollo de competencias básicas y profesionales que se fundamentan en el pensamiento práctico. Así, la teoría se convierte en una herramienta privilegiada para comprender, entender y diseñar la práctica. En los principios pedagógicos del Modelo de la UNAE se enfatiza la importancia de un aprendizaje basado en problemas, casos, situaciones y proyectos que motiven al estudiantado a reflexionar y a plantear estrategias activas que transformen los contextos socioeducativos.

En este sentido, el Modelo Pedagógico de la UNAE resalta la importancia del componente práctico del currículo de formación, cuyo 40 \% está constituido por actividades prácticas en contextos reales que permiten articular dos momentos fundamentales en la formación profesional: la teorización de la práctica y la experimentación de la teoría.

Bajo el principio de pertinencia y reciprocidad, el estudiantado de la UNAE, desde el primer ciclo se inserta a las prácticas en las instituciones educativas participantes, con el objetivo principal de llevar a cabo un proceso organizado de investigación a partir de las necesidades de la institución educativa y mejorar las prácticas educativas de todos los actores clave. De este modo, la práctica preprofesional se convierte en el centro de convergencia de la teoría y las vivencias desarrolladas en las realidades socioeducativas.

Entonces, surge la pregunta: ¿cómo se llega a este punto de convergencia? ¿Es suficiente asistir a las aulas universitarias, recibir la teoría y luego aplicar en la escuela los contenidos adquiridos? Para despejar estas interrogantes es necesario desarrollar ideas que se acerquen a conocer cómo se desenvuelve el pensamiento práctico a partir del Modelo Pedagógico y el Modelo de Prácticas Preprofesionales de la UNAE, y cómo estos entran en diálogo con la dinámica de la práctica preprofesional.

Las tutoras y los tutores académicos juegan el papel esencial de acompañar a reflexionar para generar conocimiento. Para entender esta dinámica, partimos de las premisas de Schön (1998), quien denomina "repertorio" a las vivencias de la práctica, útiles para comprender y aprender (imágenes, comprensiones y actuaciones). En este sentido, en cada 
una de las situaciones que vive el alumnado, el autor hace uso de ese repertorio, y es a través de él que la situación es reconocida y modificable (conocimiento en acción). Este es el punto de partida para provocar lo que Schön llama "conversación reflexiva con la situación", en la que el estudiantado puede cuestionar o no el uso que ha hecho de ese "repertorio".

Una de las estrategias usadas por tutores y tutoras académicas como método para reflexionar sobre la práctica es crear espacios de intercambio donde cada estudiante narra la situación o situaciones que más le ha llamado la atención o la que más dificultad, asombro y/o tensión le provocó. Para Cladinin y Conelly, “la práctica reflexiva implica [...] la evocación de imágenes desde una historia, desde una narrativa de experiencia, de tal forma que la imagen sea, entonces, capaz de guiarlos para dar sentido a futuras situaciones" (En Clarà y Mauri, 2010, p.135). Así, las imágenes constituyen formas de conceptualizar las situaciones desde cada experiencia personal adquirida en la escuela, y enriquecen la relación que se establece entre las experiencias (práctica) y la formación teórica; de esta forma, las acciones que modifican las situaciones parten de un entramado de imágenes, mapas y artefactos, los mismos que componen el conocimiento práctico estudiantil. En consecuencia, la relevancia del prácticum en la formación del pensamiento práctico es parte de ese punto de convergencia entre la teoría y la práctica del que se habló al inicio y que da sentido al principio de "teorizar la práctica y experimentar la teoría".

Según Pérez Gómez et al. (2015) "teorizar la práctica debería implicar la reflexión del docente sobre su propia práctica, sobre su propia forma de actuar, a la luz de las experiencias educativas más relevantes y de los resultados de la investigación educativa más consistentes" (p. 20). En la UNAE, el aprender en y para la escuela es el aprender en y para la práctica docente; la escuela es un contexto enriquecedor, donde el estudiantado aprende a partir de las necesidades, problemáticas y situaciones que emergen desde su cotidianidad, siendo el punto de partida de un proceso organizado de investigación, que es trascendental en el desarrollo de competencias que permiten desempeñarse en el campo profesional. En este sentido, la investigación en la formación docente también promueve procesos reflexivos a partir del diagnóstico, el diseño, la planificación, la evaluación y, por último, las propuestas de mejora e innovación basadas en la situación diagnosticada.

\subsection{Los entornos virtuales frente a la educación presencial}

La repentina transición de la educación presencial al uso de entornos virtuales a causa del Covid-19 significó un desafío para la mayoría de las instituciones educativas, docentes y 
estudiantes; unas se mostraron más preparadas que otras debido a que ya habían incorporado el uso de las TIC en la educación, lo que propició la implementación de prácticas innovadoras para abordar los procesos de enseñanza-aprendizaje. Para Bustos y Coll (2010), el uso de las TIC en educación mediante las aulas virtuales y el aprendizaje en línea ha ampliado los sistemas de interconexión e intercomunicación. De igual manera, Abreu (2020) describe a la educación en línea como una forma de aprender que permite la interacción entre docentes y estudiantes desde cualquier lugar fuera del campus, siempre y cuando tenga electricidad, una computadora con dispositivos de audio y cámara, y acceso a internet.

El contexto de pandemia impulsó el uso de los entornos virtuales de enseñanza que se pusieron a disposición de docentes y estudiantes de todos los niveles para que puedan continuar con los procesos educativos y no tengan que esperar a que se pueda volver a la modalidad presencial. Poniendo el uso de la tecnología en primera línea dentro del área educativa, Arce y Guiller (2015) señalan que la tecnología debe estar al servicio de los fines educativos, permitiendo así que, ante la enseñanza en línea, el profesorado sea quienes decidan cómo enseñar, es decir, que tengan la libertad de tomar decisiones pedagógicas y didácticas que superen los modelos educativos donde el personal docente sean meros transmisores de información. Con un planteamiento similar, Expósito explica que la educación en línea debe pasar por una "adecuación de la docencia y un ajuste de los procesos y materiales empleados" (2020, p. 7) para evitar que el uso de herramientas diferentes de comunicación suponga, simplemente, el uso de una videoconferencia o plataformas virtuales que apenas son herramientas complementarias de comunicación.

Así, podrían considerarse dentro del uso adecuado de los entornos virtuales:

Diseños que centran sus estrategias en trabajos de construcción colectiva de conocimiento de los estudiantes, en espacios que favorecen la interacción y el intercambio de experiencias y saberes, en torno a ejes de problematización y discusión; producciones colaborativas en diferentes lenguajes y formatos hipermediales que tienen lógicas y modos de construcción particulares; uso de recursos y de espacios en la web que trascienden las aulas virtuales; promoción de experiencias y prácticas en entornos tecnológicos que representan desafíos para los estudiantes, donde deben desplegar nuevas estrategias, construir nuevos significados para buscar, participar, investigar, producir, colaborar, construir con otros. (Arce y Guiller, 2015, p. 5) 
De igual manera, como indicadores para identificar buenas prácticas en entornos virtuales García Martínez et al. (2015), mencionan que el docente debe usar diferentes recursos para mediar el aprendizaje de los contenidos, espacios como foros, plataformas para mensajería instantánea, correo electrónico y otros que permitan la comunicación entre estudiantes y su docente; asimismo, resaltan la idea de que el personal docente debe respetar las diferentes formas de aprender del estudiantado.

Si bien, estas propuestas son muy interesantes, Suáres Álvarez et al. (2020) concluyen que debería existir inversión/planes de alfabetización digital dirigidos al profesorado, que le permita usar las TIC de manera eficaz, además que no desmerecen la búsqueda de mejora continua de la infraestructura tecnológica de las instituciones educativas, sin descuidar el carácter pedagógico y los modelos didácticos a aplicarse.

Por otro lado, la tan anhelada educación presencial "ha constituido la base de la transmisión de conocimiento durante siglos, fundamentalmente aporta el hecho enriquecedor del contacto socializador, de la expresión corporal de sensaciones, anhelos y emociones, imposibles de emular a distancia" (Martínez, 2017, p. 112). En este sentido, llevar a cabo el complejo proceso de enseñanza-aprendizaje in situ, pone al alcance de las y los estudiantes, la oportunidad de desarrollar habilidades sociales y emocionales, además de adquirir destrezas y valores que les enseñen a convivir en sociedad, haciendo que la experiencia de aprender sea más enriquecedora. Sobre la modalidad presencial se puede encontrar más ventajas que desventajas, algunas de ellas son:

- Proporciona una relación cercana con docentes y sus pares, lo que podría suponer una mayor motivación, ya que, como es conocido, esta modalidad consiste en brindar espacios de asistencia regular a las aulas dentro de los establecimientos escolares.

- La presencialidad proporciona más empatía entre estudiantes y docentes, lo que brinda mayor oportunidad de que estos últimos conozcan cada individualidad y necesidad para, con base en ellas, mediar el aprendizaje.

Sin duda, la modalidad presencial podría poner a disposición de profesores y alumnos un amplio abanico de opciones en cuanto a metodologías que, entre otras, podrían ser: clase magistral, estudio de caso, aprendizaje por proyectos, flipped classroom o aula invertida, exposiciones orales, participación en seminarios, conferencias, trabajos grupales.

A manera de conclusión sobre el presente bloque, se puede acotar que la educación en línea merece una planificación estricta, que va desde lo metodológico, la infraestructura, la 
dotación de un equipo básico de computación, hasta la disponibilidad de un espacio físico para educadores y educandos; es decir, para hacer frente a la modalidad virtual se deben buscar las mejores formas de solventar el proceso de enseñanza-aprendizaje mientras dure la emergencia sanitaria.

\section{Metodología}

La práctica preprofesional juega un rol muy importante en la formación de docentes de la carrera de Educación Especial. Por lo tanto, las condiciones brindadas a cada estudiante para la experimentación y el desarrollo del pensamiento práctico dependerán de las condiciones en las que se desarrolle la práctica preprofesional y la reflexión. Esto significa que desde la modalidad presencial, todo el estudiantado tiene contacto directo con la realidad educativa: estudiantes, docentes y padres de familia, desarrollándose procesos de aprendizaje en y para la acción; pero, ¿qué sucede en la práctica virtual? Desde la experiencia desarrollada en la carrera de Educación Especial, a partir de la pandemia, la práctica preprofesional presentó algunas particularidades, razón por la cual nace la iniciativa de realizar un proceso de validación de esta experiencia en la formación inicial de docentes.

\subsection{Enfoque}

El proceso de validación de la práctica preprofesional en la modalidad virtual se realizó a través de una investigación cualitativa, con un alcance descriptivo a través del método participativo, en el que intervinieron actores clave: estudiantes, tutores y tutoras profesionales y académicos. Para López (2007), en el método participativo intervienen procesos que involucran activamente a participantes que contribuyen en la transformación social y la generación de nuevos conocimientos.

\subsection{Unidad de análisis}

En esta investigación participaron 377 estudiantes matriculados en la carrera en el periodo académico IS-2020, 9 tutoras y tutores académicos responsables de la práctica preprofesional y 160 tutoras y tutores profesionales que laboran en las escuelas de prácticas, con quienes se socializaron los objetivos de este proyecto y se procedió a la firma del consentimiento informado y al compromiso de confidencialidad. Este documento permitió utilizar la información recogida para fines académicos como la elaboración de un documento orientador para la práctica preprofesional del siguiente ciclo y la publicación de los resultados. 
La muestra fue intencionada, considerando la totalidad de docentes de la carrera de Educación Especial de la UNAE (tutoras y tutores académicos) que se encargan de las prácticas preprofesionales de todos los ciclos, estudiantes de la carrera de Educación Especial y docentes de las escuelas de prácticas (tutoras y tutores profesionales).

\subsection{Técnicas de recolección de datos}

En el proceso de recolección de información se aplicaron las siguientes técnicas de investigación cualitativa: cuestionarios en línea, los mismos que fueron validados a través de un juicio de expertos, revisión documental y grupo de discusión. Los cuestionarios en línea fueron aplicados a estudiantes y tutores profesionales; se elaboró un cuestionario con preguntas abiertas en la plataforma Google Forms en el que se tomaron en cuenta los tres momentos de la práctica preprofesional en la modalidad virtual: coordinación, acompañamiento y resultados.

La coordinación incluye los procesos de gestión y organización de la práctica de la tutora o tutor académico con la institución educativa y con el estudiantado.

El acompañamiento se refiere a los procesos que la tutora o tutor académico implementa en la modalidad virtual para el cumplimiento del plan de práctica, el mismo que está articulado al núcleo problémico y al eje integrador de cada ciclo; la coordinación con tutoras y tutores profesionales, y la creación de espacios para la reflexión de la práctica y la experimentación de la teoría con el apoyo del docente de Cátedra Integradora.

Los resultados reflejan los aprendizajes logrados en la práctica preprofesional en modalidad virtual, los mismos que guardan correspondencia con los resultados de aprendizaje contemplados en la planificación microcurricular de la carrera.

Se realizó la revisión documental del informe de prácticas elaborado al término del periodo IS-2020 por cada tutora o tutor académico, y el grupo de discusión. En este informe constan datos sobre el cumplimiento del plan de prácticas, actividades desarrolladas y resultados de aprendizaje alcanzados en correspondencia con el núcleo problémico y eje integrador de cada uno de los ciclos.

Finalmente, el grupo de discusión -desarrollado con nueve tutoras y tutores académicos- analizó las fortalezas y dificultades presentadas en la práctica preprofesional en la modalidad virtual. Este espacio promovió el análisis y la reflexión sobre la importancia de los ambientes virtuales para el aprendizaje y la participación de futuros docentes. 


\subsection{Procesamiento de análisis}

El análisis de datos se realizó siguiendo el análisis de contenido; Bardin define a este proceso como "un conjunto de técnicas de análisis de las comunicaciones utilizando procedimientos sistemáticos y objetivos de descripción del contenido de mensajes" (2002, p. 29). La fase de análisis propiamente dicha se inició estableciendo el sistema de categorización y codificación de la información, en el que se consideraron los momentos de la práctica preprofesional con sus respectivas categorías, las mismas que guardan correspondencia con el rol que desempeña cada actor de acuerdo con el Modelo de Prácticas Preprofesionales de la UNAE. Una vez establecido el sistema de categorización y codificación, se organizó la información de los datos obtenidos de los actores clave: estudiantes, tutoras y tutores académicos, tutoras y tutores profesionales, con relación a la práctica preprofesional de la carrera de Educación Especial, para lo cual se utilizó el programa informático Atlas Ti versión 9.

En la construcción de las categorías también se consideraron los tres momentos de la práctica preprofesional: coordinación, acompañamiento y resultados. La información fue organizada sistemáticamente a través de las subcategorías que se detallan a continuación:

En la categoría coordinación se identificaron las subcategorías:

- Coordinación de la práctica preprofesional (CPP)

- Distribución de parejas y/o tríos pedagógicos (OPTP)

- Coordinación con tutoras y tutores profesionales (CTP)

- Socialización del plan de práctica (SPNP)

En la categoría acompañamiento, se encontraron las subcategorías:

- Experimentación de la teoría a partir de la práctica (ETPP)

- Espacios y oportunidades para la reflexión (EOR)

- Organización de actividades (OA)

- Tutorización de la práctica preprofesional (TPP)

- Acompañamiento del tutor o tutora profesional (ATP)

En la categoría resultados se establecieron las subcategorías:

- Elaboración del PIENSA (EP)

- Resultados de aprendizaje (RA) 
Una vez establecidas las categorías y subcategorías, se analizó la información empleando los datos obtenidos de tutoras y tutores académicos, tutoras y tutores profesionales y estudiantes, a quienes se les aplicó los instrumentos ya mencionados. Debido a la situación de la pandemia, la aplicación del cuestionario a estudiantes y a personas tutoras profesionales se realizó a través de Google Forms. Con las tutoras y los tutores académicos se aplicaron dos técnicas: revisión documental (informe de prácticas) y grupo de discusión, el mismo que se desarrolló a través del sistema de videoconferencia Zoom.

\section{Resultados}

Los resultados del cuestionario en línea se encuentran organizados en función de los tres actores de la práctica preprofesional (PP): estudiantes, tutores profesionales y tutores académicos.

\subsection{Estudiantes}

\subsubsection{Momento 1: Coordinación}

Este primer momento corresponde a la subcategoría coordinación de la práctica preprofesional (CPP) relacionada con la explicación del tutor académico sobre la PP en la modalidad virtual; la articulación de la PP con el eje integrador y núcleo problémico del ciclo, la coordinación de la práctica preprofesional del tutor académico con tutores profesionales y estudiantes, la organización de los grupos de trabajo (parejas, tríos, etcétera), correspondiente a la subcategoría distribución de parejas y tríos pedagógicos (OPTP) se realizó en función de la realidad de cada institución educativa, es decir, tomando en cuenta el número de estudiantes y paralelos con los que cuenta con escuela, la coordinación con los tutores y las tutoras profesionales (CTP) se realizó mediante correo electrónico para los encuentros sincrónicos con las personas tutoras profesionales y/o estudiantes. Finalmente, la socialización del plan de práctica (SPNP) se realizó en la primera reunión que se mantuvo con los tutores y tutoras académicas (Ver figura 1). 
Figura 1

Coordinación de la práctica preprofesional, primer semestre 2020

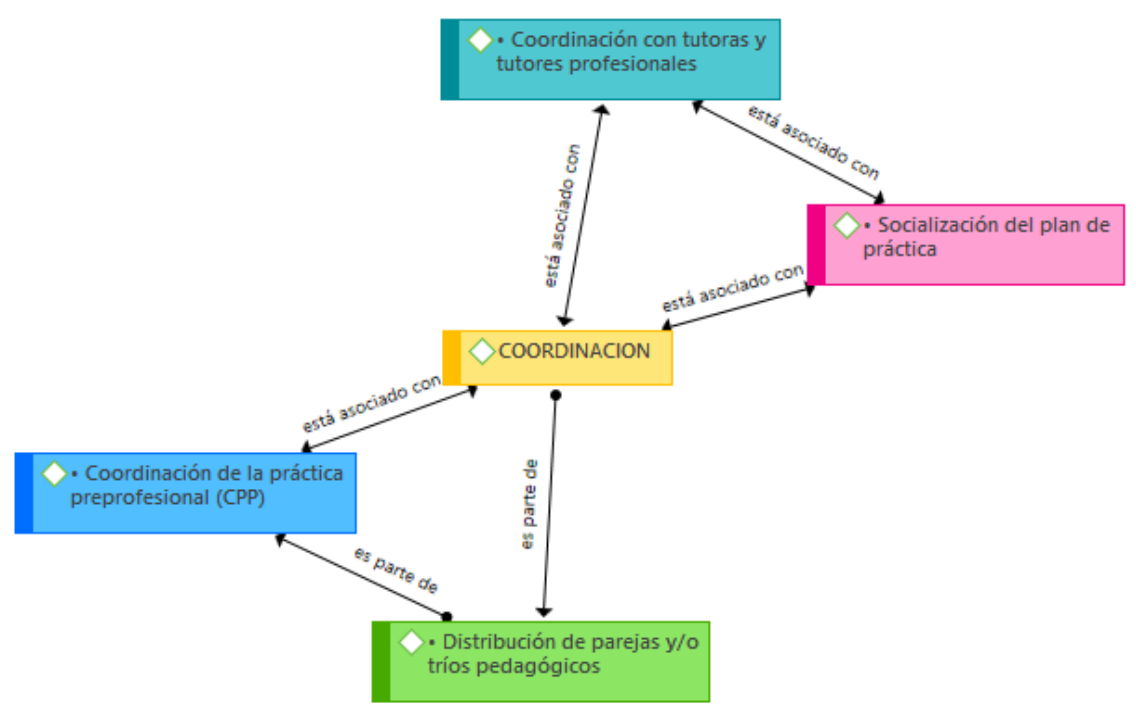

Fuente: Elaboración propia, con información obtenida de los actores de la práctica profesional, 2020

El estudiantado manifiesta que estos procesos se desarrollaron de manera "muy satisfactoria"; sin embargo, en algunos casos, en el espacio abierto de opiniones dentro del cuestionario, algunos estudiantes señalan que existieron personas tutoras académicos que no coordinaron adecuadamente con la institución educativa, situación que generó dificultades al momento de cumplir las actividades del plan de práctica y coordinar con tutores y tutoras profesionales. "Nuestro profe solo envió un correo y no se reunió con los profes de las escuelas, entonces ellos no sabían qué teníamos que hacer" (E56). "Nuestro profe nos envió a nosotros a presentar el plan de práctica a la tutora" (E86).

En los casos de instituciones educativas con pocas aulas, se tuvo que ajustar la distribución del alumnado para que todos pudieran participar en los encuentros sincrónicos; así, en algunos casos, los grupos de estudiantes se organizaron con cuatro o cinco integrantes. "[...] Nos tocó organizarnos en grupos de cuatro estudiantes porque la escuela tenía pocas aulas, no pudimos trabajar en pareja pedagógica" (E45).

Por otro lado, no todas las personas tutoras profesionales asistieron al encuentro sincrónico de inducción de la PP en la que se socializó el plan y se establecieron los acuerdos con la institución sobre las modalidades de trabajo. Así, al momento de la ejecución, la escasa información que tenían esta población provocó que se ejecuten actividades que no correspondían al plan de prácticas: "Mi tutora no asistió a la inducción, no sabía qué tenía que hacer con nosotros y nos pedía que hagamos materia para sus clases" (E287). 


\subsubsection{Momento 2: Acompañamiento}

El proceso de acompañamiento (Figura 2) presentó ciertas limitaciones, una de ellas fue la afectación que sufrió por el recorte presupuestario ${ }^{4}$, por lo que la mayor parte de docentes se desempeñó a medio tiempo, En ciclos anteriores, la mayoría de tutoras y tutores académicos realizaban el acompañamiento a diario; en cambio, en este ciclo, algunas personas tutoras académicas destinaron a esta actividad solamente de tres a cinco horas semanales, y tuvieron que dedicar tiempo extra para solventar situaciones que se presentaron en las escuelas. "Cuando estábamos en la escuela, los profes nos acompañaban todos los días, ahora en clases virtuales solo les contamos lo que sucede y los profes nos dan indicaciones" (E57). "En mi caso, cuando yo tenía práctica, mi profe estaba en clases con otro curso y no podía asistir a los encuentros sincrónicos" (E248).

\section{Figura 2}

Proceso de acompañamiento de la práctica preprofesional, primer semestre 2020.

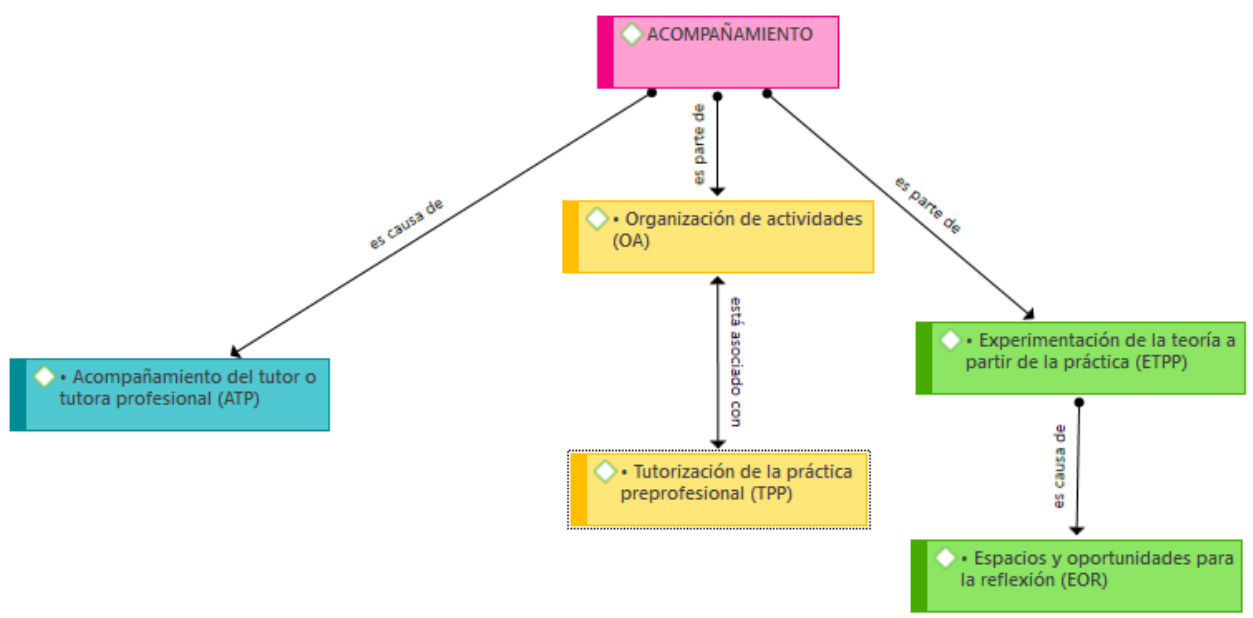

Fuente: Elaboración propia, con información obtenida de los actores de la práctica profesional, 2020.

Pese a todas las dificultades, las tutoras y tutores académicos lograron coordinar las actividades con las tutoras y tutores profesionales y autoridades de las escuelas a través de correo electrónico y WhatsApp; y monitorear las actividades que el estudiantado debía cumplir en el periodo de las PP.

\footnotetext{
${ }^{4}$ En mayo de 2020, el Gobierno anunció disminuciones de alrededor del $10 \%$ del presupuesto de las Universidades públicas del Ecuador, lo que supuso la supresión de algunos contratos.
} 
En este indiciador, el alumnado afirma que la comunicación establecida entre profesionales y académicos fue muy satisfactoria, ya que se aprovecharon los encuentros sincrónicos de las escuelas para propiciar espacios de orientación y retroalimentación de la PP, así como también la creación de grupos de chat en WhatsApp.

La colaboración, en general, de las personas tutoras profesionales fue satisfactoria; sin embargo, algunas escuelas no facilitaron los documentos académicos, tales como: Proyecto Educativo Institucional (PEI), Plan de Unidad Didáctica (PUD) y Código de Convivencia. Algunos tutores profesionales manifestaron que la virtualidad limitó la reflexión de la realidad educativa, ya que no todo el estudiantado pudo participar en los encuentros sincrónicos: "Los tutores de la escuela no enviaban los enlaces para entrar a las clases" (E123). "Mi tutora me dejaba en la sala de espera y me aceptaba cuando faltaba cinco minutos para que se termine la clase" (E38). En efecto, ciertos tutores profesionales no enviaron los enlaces para que se conecte el grupo de practicantes; otros no realizaron encuentros sincrónicos debido a que no disponían de conexión a internet. Y, por último, en las escuelas de educación especializada no se realizaron encuentros sincrónicos debido a las condiciones de discapacidad que presenta la mayoría de personas matriculadas.

Algunos estudiantes manifiestan que en este ciclo no se evidenció la coordinación de la pareja pedagógica académica ${ }^{5}$, razón por la cual, la experimentación de la teoría a partir de la PP virtual no se cumplió: "Antes el profe de Cátedra nos ayudaba con el profe de Práctica en la reflexión para construir el PIENSA, ahora cada profe da su clase por separado" (E9). En algunos ciclos, el profesorado de Cátedra, Práctica e Investigación coordinó el PIENSA en la etapa final, solamente para la entrega del producto. Algunos estudiantes señalaron que:

- "El profe en las horas de prácticas nos dio clase de su materia" (E4).

- "Le pedimos a la profe que nos ayude, pero la profe de la escuela no nos ayudó" (E203).

- "No supimos qué hacer concretamente y los profes de la escuela nos pidieron que hagamos material para sus clases" (E189).

- "Los profes de las escuelas se molestaban cada vez que les pedíamos algo, no querían ayudarnos" (E12).

- "Con esta práctica no se aprende" (E356).

\footnotetext{
${ }^{5}$ La conforman los docentes de las asignaturas de Aproximación/Exploración Diagnóstica y Cátedra Integradora, que a partir del principio de experimentar la teoría y teorizar la práctica deben juntos planificar, desarrollar y evaluar los procesos formativos, además de guiar y brindar espacios de reflexión desde las vivencias de la práctica.
} 


\subsubsection{Momento 3: Resultados}

Los resultados obtenidos de la voz de todo el estudiantado que participó en este estudio demuestran que la PP virtual debe ser revisada y replanteada, ya que las actividades contempladas en el plan fueron inicialmente diseñadas para la modalidad presencial, pero por la emergencia sanitaria tuvieron que cambiarse a la modalidad virtual, produciendo ciertas deficiencias académicas en la mejora de la práctica docente (Ver Figura 3).

Figura 3

Resultados obtenidos de la práctica preprofesional, primer semestre 2020.

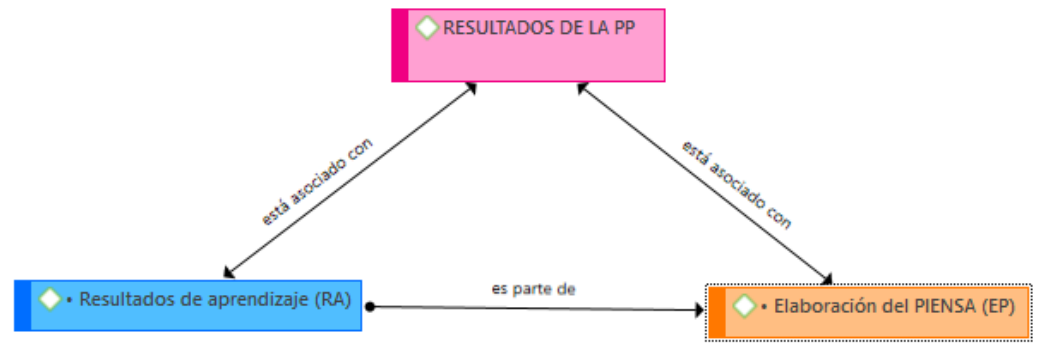

Fuente: Elaboración propia, con información obtenida de los actores de la práctica profesional, 2020.

En su mayoría, el alumnado reporta que se lograron aprendizajes; sin embargo, existen algunos indicadores que llaman la atención, por ejemplo, el proceso de elaboración del PIENSA y el informe de las experiencias vividas en la modalidad virtual. "En las prácticas presenciales podíamos tener relación directa con la profe y los niños". "Para construir el PIENSA tuvimos que conversar con la profe para que ella nos cuente cómo es el niño y nosotros solo le vimos en una clase sincrónica" (E168). El resultado de estos dos últimos indicadores nos lleva a repensar los procesos implementados para el cumplimiento de los productos de las PP.

Dentro del cuestionario aplicado se habilitó un espacio para aspectos que no fueron considerados en las preguntas planteadas, del cual se obtuvieron los siguientes testimonios:

- "El profe de la escuela nunca apareció, a pesar de que le mandamos correos y mensajes al WhatsApp" (E217).

- "No supimos cómo hacer el PIENSA porque no estuvimos en la escuela y así no podemos identificar las necesidades".

- "Para el PIENSA solo nos basamos en lo que los profes nos contaron en la entrevista" (E38). 


\subsection{Tutoras y tutores académicos}

La información de las tutoras y los tutores académicos fue obtenida de la revisión documental del informe de práctica preprofesional y del grupo de discusión. Como se manifestó anteriormente, las personas tutoras académicos tuvieron muy poco tiempo asignado para las PP, situación que llevó al claustro docente a organizar en tiempo extra el proceso de las PP en cada ciclo, además, muchos de ellos asumieron por primera vez esta actividad académica.

\subsubsection{Momento 1: Coordinación}

La coordinación de la PP se realizó a partir de un distributivo realizado por cada docente que se desempeñó como miembro del equipo de gestión de las prácticas, representante de la carrera de Educación Especial. A partir de esta organización, cada docente responsable de la práctica estableció contacto y coordinó con la institución educativa.

De manera general, el proceso de coordinación se realizó de forma satisfactoria; no obstante, existen dos indicadores que llaman la atención: la colaboración de tutores profesionales y autoridades de las escuelas y la falta de coordinación de encuentros sincrónicos para que cada practicante participe. Esta situación se debió a varios factores: a) bajo la modalidad virtual, los tutores profesionales estuvieron desarrollando encuentros sincrónicos en diferentes horarios, por lo que muchos de ellos no coincidieron con los horarios de PP; b) poca disposición de los tutores profesionales para que el estudiantado participen, ya que se sintieron observados y/o evaluados.

\subsubsection{Momento 2: Acompañamiento}

El acompañamiento consideró la organización de actividades (OA) que se realizó a través de encuentros sincrónicos y mediante correo electrónico, aula virtual y WhatsApp, con la finalidad de solventar inquietudes o dificultades presentadas en el proceso de las PP tanto con estudiantes como con tutores profesionales. Con respecto a la experimentación de la teoría a partir de la práctica (ETPP) y a los Espacios y oportunidades para la reflexión (EOR) existieron algunas limitaciones para la coordinación con parte del personal docente de Cátedra Integradora e Investigación, pero para la elaboración del PIENSA se coordinaron de la mejor forma los procesos tanto del diseño como de presentación de los trabajos. "Nos tocó trabajar fuera de horario para revisar los trabajos de los alumnos" (TA3). 
Con relación a la tutorización de la práctica preprofesional (TPP) y al acompañamiento del tutor o tutora profesional (ATP), la mayoría de tutores académicos manifiestan que el acompañamiento se desarrolló "muy satisfactoriamente", salvo dos indicadores en los que se obtuvieron resultados "satisfactorios": a) conectividad inestable, por lo que no todos sus estudiantes pudieron participar en los encuentros sincrónicos, y b) la PP virtual no permitió cumplir con los ejes vertebradores de la PP contemplados en el Modelo de la UNAE: ayudar, acompañar y experimentar; las actividades se limitaron al desarrollo de material didáctico, observaciones y, en escasas ocasiones, el desarrollo de clases planificadas. "En modalidad virtual es muy complicado que todos los estudiantes puedan participar, muchos de ellos ni a clases asisten" (TA7).

\subsubsection{Momento 3: Resultados}

Los resultados de aprendizaje (RA) alcanzados en la PP han propiciado la reflexión, análisis y reinvención de los procesos educativos en la modalidad virtual. Estas condiciones generaron otros modos de aprender y de acercarse a la realidad educativa, así como también promovieron la innovación en las prácticas docentes para responder a la diversidad de estudiantes y que, de alguna manera, se eliminen las barreras para el aprendizaje y la participación.

Los resultados alcanzados demuestran que la virtualidad ha limitado el proceso formativo de la gran mayoría de estudiantes. Al no tener contacto directo con el entorno educativo, considerado como un espacio privilegiado para aprender en y para la acción, gran parte de tutores académicos tuvieron que diseñar nuevas formas para cumplir con la PP, y su grupo de estudiantes realizó actividades de acuerdo a la dinámica de la escuela que, en muchos casos, estuvo alejada del plan de PP diseñado inicialmente. Otro aspecto para destacar es la limitada participación de estudiantes en los encuentros sincrónicos y el desarrollo o intervención en las clases organizadas por las tutoras y tutores profesionales.

Con respecto a los resultados obtenidos en la revisión documental del informe de la práctica, los tutores académicos afirman que la práctica preprofesional en modalidad virtual promueve otras formas de aprendizaje en las que se involucran diversas estrategias para el desarrollo del pensamiento práctico. Con respecto a la elaboración del PIENSA (EP) "La práctica virtual se convirtió en una oportunidad para el aprendizaje porque mis estudiantes aprendieron a utilizar recursos tecnológicos" (TA9). Si bien es cierto, la modalidad virtual no permite el contacto directo con la comunidad educativa de la escuela, esta ha propiciado 
espacios de reflexión sobre las diversas formas de educación en otros contextos. En este sentido, se promovieron espacios en los que el estudiantado y las tutoras y tutores profesionales intercambiaron vivencias y experiencias de buenas prácticas docentes. Se diseñaron propuestas innovadoras inclusivas que dieron respuesta a la situación educativa actual, mediante la organización de espacios de encuentro para la tutorización y acompañamiento a estudiantes con necesidades educativas especiales. Se elaboraron guías metodológicas dirigidas a las familias, las mismas que contienen actividades cotidianas que promueven el desarrollo de las destrezas específicas según la necesidad del alumnado. Se implementaron plataformas de atención a la diversidad, lo que facilitó el trabajo de practicantes y docentes en modalidad virtual. Todas estas actividades dieron respuesta a las limitaciones de conectividad que se presentaron con el alumnado de las escuelas de prácticas. Así, se cumplió la mayor parte de actividades formuladas en el plan de práctica, las mismas que se encuentran establecidas en la planificación microcurricular de la carrera.

Por otro lado, el profesorado afirma que la virtualidad aleja a sus practicantes de las aulas, limitando la potencialidad de la práctica -eje vertebrador de la formación de futuros docentes -, ya que en esta modalidad no se establece una relación directa con los agentes educativos, ni se observa la realidad de la escuela. Además, la mayoría de encuentros sincrónicos de las escuelas no coincidió con el horario de clases de los practicantes, por lo estos no pudieron participar en su totalidad de las clases virtuales organizadas por sus tutores profesionales, y se limitaron a la recopilación de información a través de entrevistas a docentes y a la revisión documental de las planificaciones curriculares, comprometiendo los objetivos de aprendizaje según el ciclo.

Para el grupo de discusión se contó con la participación del profesorado de la carrera (tutoras y tutores académicos) que dio cuenta de la importancia de la práctica preprofesional en modalidad presencial, y afirma que "la riqueza de la experiencia educativa está en el contacto y la vivencia diaria en los contextos educativos" (TA4). La modalidad virtual permitió plantear recursos tecnológicos y propuestas educativas de atención a la diversidad, pese a que muchos practicantes no tuvieron contacto directo con estudiantes de las escuelas, situación que limitó la identificación, cuestionamiento, análisis y reflexión de la práctica docente y los procesos de transformación del sistema educativo ecuatoriano.

A pesar de las debilidades identificadas, varios tutores académicos afirman que se pueden mejorar los procesos de relación con las instituciones educativas, especialmente con 
tutores profesionales, quienes se convierten en coformadores del proceso y acompañantes de las prácticas en las escuelas.

\subsection{Tutoras y tutores profesionales}

\subsubsection{Momento 1: Coordinación}

Como parte del protocolo de organización de la PP, las instituciones educativas recibieron un proceso de inducción en el que se socializó el plan de prácticas y se coordinaron los procesos en común acuerdo con tutores y tutoras profesionales, ya que debido al plan de contingencia emitido por el MinEduc este grupo de docentes, solicitó a la UNAE participar como apoyo en los procesos educativos; es decir, en la elaboración del material educativo y diseño de propuestas educativas para la atención a la diversidad.

El proceso de coordinación se desarrolló "satisfactoriamente"; gran parte de las tutoras y tutores profesionales afirman que no todos los tutores y tutoras académicos coordinaron adecuadamente las prácticas, incluso algunos de ellos no realizaron la inducción, razón por la que tanto estudiantes como docentes no tenían las directrices claras de las actividades a desarrollarse. "El profesor encargado de la práctica no se reunió conmigo para explicarme cómo se iban a desarrollar las actividades" (TP8).

En cuanto al proceso de comunicación manifiestan que "los docentes de la UNAE no se involucraron como en otros ciclos". "Algunos tutores de las UNAE nos dieron su número de WhatsApp para conversar rápidamente" (TP4). Por situaciones de conectividad y por la realidad de cada escuela existieron limitaciones para el involucramiento de practicantes en la dinámica educativa, y no se logró tener contacto directo con estudiantes de las aulas asignadas, especialmente en las escuelas de educación especializada.

\subsubsection{Momento 2: Acompañamiento}

Pese a las condiciones complejas en las que se desarrollaron las clases, la mayor parte del profesorado de las escuelas mostraron disposición para colaborar con el proceso de prácticas profesionales de la carrera. Los procesos de acompañamiento se desarrollaron satisfactoriamente, ya que existió coordinación entre tutoras y tutores profesionales y practicantes. "Nos pusimos de acuerdo con los practicantes para que ellos me apoyen en las clases" (TP67). No todo el alumnado pudo intervenir en las actividades de la institución educativa debido a situaciones de conectividad y coordinación de actividades con los padres de familia mediante WhatsApp, esta última situación se produjo en las escuelas de educación 
especializada en las que no se desarrollaron encuentros sincrónicos, sino se coordinaban actividades semanales con las familias, quienes enviaban fotografías de las tareas realizadas.

\subsubsection{Momento 3: Resultados}

El cumplimiento de los resultados de la PP se realizó de manera "satisfactoria", pero existieron factores que impidieron que parte del estudiantado identifique las necesidades 0 problemáticas de la escuela. Esto se debió a que en la modalidad virtual no tuvieron contacto directo con estudiantes de las escuelas de prácticas, como ya se señaló anteriormente. No obstante, algunos tutores profesionales manifestaron que "las PP permitieron desarrollar nuevas estrategias pedagógicas a través de la aplicación de recursos tecnológicos" (TP3), los mismos que fueron propuestos por practicantes. Además, se demostró un compromiso e involucramiento con la realidad escolar, el desarrollo del pensamiento práctico y la apertura e implicación con el proceso de enseñanza-aprendizaje en el aula.

Asimismo, las tutoras y tutores sugieren que el alumnado de esta carrera adquiera un lenguaje técnico con respecto a la discapacidad e inclusión, que elabore material innovador para el aprendizaje y atención a la diversidad, y que promueva actividades lúdicas para la atención a la diversidad en la modalidad virtual. Por otro lado, recomiendan que se debe mejorar la distribución de acciones de cada estudiante dentro de la pareja pedagógica, coordinar la dedicación y entrega en las actividades realizadas en las PP y tomar la iniciativa en las actividades pedagógicas, este aspecto se encuentra a cargo delas tutoras y tutores académicos.

\section{Discusión}

Una vez obtenidos los resultados se presenta la discusión con base en la pregunta de investigación: ¿Cómo la práctica preprofesional en modalidad virtual contribuye al aprendizaje de las y los docentes en formación de la carrera de Educación Especial? Para responder esta interrogante es importante considerar que la práctica pedagógica incluye un conjunto de acciones que contribuyen al proceso de formación integral y al desarrollo del pensamiento práctico del alumnado en educación superior.

Como se desprende de los resultados presentados en este documento, existen algunas inconsistencias en la práctica preprofesional en modalidad virtual, elementos que influyen en los procesos de enseñanza-aprendizaje. Terranova Ruiz et al. (2015) refieren que la práctica preprofesional aporta a la formación integral mediante la construcción del conocimiento que 
se desarrolla con el saber, el saber hacer y el saber actuar en un contexto que acerca a la realidad educativa. De este modo, la práctica preprofesional en modalidad presencial complementa la formación académica que recibe el estudiantado en las aulas, aportando al desarrollo de competencias que los preparan para el desempeño profesional; de manera especial, en la UNAE, a partir del espacio de reflexión que se genera después del trabajo in situ, los aprendizajes adquiridos se integran al bagaje académico a través de la reflexión sobre las prácticas con las tutoras y tutores profesionales, y desde las propias acciones de las y los estudiantes. De manera general, desde cada proyecto institucional, las prácticas preprofesionales se convierten en un elemento que merece planificación, acompañamiento y seguimiento riguroso, con la finalidad de propiciar un contexto donde se puedan integrar la teoría y la práctica mediante la identificación de situaciones problémicas, necesidades y prácticas exitosas.

Analizando desde otra perspectiva, Zabalza (2017) abre un debate entre la enseñanza online y la presencial, y plantea la necesidad de establecer comunidades de aprendizaje. Desde la experiencia de la UNAE, el vínculo y organización que se da entre estudiantes, a partir del intercambio de experiencias vividas en la escuela de prácticas preprofesionales y en los espacios de reflexión que se desarrollan en la universidad, se convierte en uno de los pilares generadores del aprendizaje. De ahí la importancia de que el alumnado se organice en parejas o tríos pedagógicos y sean acompañados por su tutora o tutor académico, ya que estos encuentros abren espacios para el debate y la reflexión sobre el contexto educativo y la práctica docente. En conclusión, es la relación entre docentes, contenidos, situaciones de la práctica y estudiantes, lo que propicia el contexto para que se generen los conocimientos. En esta línea, se ha demostrado que los encuentros presenciales permiten un mayor y más eficaz acercamiento a la realidad cotidiana, situación que no se llega a dar en la modalidad virtual por la limitación de horarios de docentes y estudiantes, o por problemas de conectividad. No obstante, estas dos modalidades propician el desarrollo de comunidades en las que todos se involucran para la búsqueda y solución de las problemáticas encontradas en las escuelas de prácticas.

Pese a lo repentino que fue para todos adaptar las tareas educativas a la modalidad virtual, con la creación de un plan de prácticas virtuales se pretendió acercar tanto a practicantes como a docentes a escenarios reales a partir de la creación de diversos contextos para así enriquecer las experiencias formativas del alumnado y que estos puedan responder a las problemáticas presentadas en las escuelas donde se desarrollan las actividades de 
prácticas en línea. Por su parte, la práctica preprofesional en modalidad presencial favorece la formación in situ, con vivencias que surgen de lo cotidiano en ambientes dinámicos y cambiantes que fomentan el desarrollo de competencias laborales que el estudiantado necesita para su desempeño profesional.

\section{Conclusiones}

La PP en modalidad virtual limita el contacto directo entre estudiantes y tutores UNAE (tutoras y tutores académicos) con la escuela de prácticas y los actores que la conforman: estudiantes, docentes y padres, lo cual disminuye el desarrollo de competencias que probablemente solo se pueden adquirir en la escuela y a través del contacto directo con las diferentes realidades.

La conectividad ha impedido la participación efectiva de estudiantes en los encuentros sincrónicos organizados por la escuela, situación que limitó a muchos practicantes a conocer e identificar las prácticas docentes implementadas en esta modalidad virtual en las escuelas de prácticas. Se evidenció la desigualdad de oportunidades que podría restringir el acceso al aprendizaje: falta de recursos tecnológicos, algunos estudiantes no disponen de computadores, ni de una conectividad adecuada para una participación activa en las prácticas, especialmente en los encuentros sincrónicos; además, se presentaron algunas barreras para la participación de estudiantes, como los horarios de los encuentros sincrónicos que no coincidieron con los horarios de las prácticas.

Debido a las condiciones mencionadas no se propiciaron espacios de reflexión de las experiencias vividas y el trabajo con la pareja pedagógica académica (docente de Aproximación Diagnóstica y Cátedra Integradora), puesto que el profesorado no contó en su distributivo docente con fondo de tiempo asignado para dicha actividad. Esto implicó que el principio de "teorizar la práctica y experimentar la teoría" se convierta en un discurso sin mayores probabilidades de cumplirse bajo estas condiciones.

Las escuelas de educación especializada no tuvieron contacto directo con estudiantes, ya que no se desarrollaron encuentros sincrónicos. Por tal razón, el alumnado de los ciclos superiores que se encuentra en la Unidad de Titulación (séptimo a noveno ciclo) no se relacionó con los procesos de atención a la discapacidad, las redes de apoyo y los programas de atención familiar.

Una ventaja de las prácticas preprofesionales en modalidad virtual es la aplicación y motivación a docentes en la búsqueda de estrategias innovadoras para el aprendizaje a través 
de recursos tecnológicos educativos. La aplicación de plataformas digitales motivó a docentes de las escuelas (tutoras y tutores profesionales) a capacitarse en tecnología educativa.

La práctica preprofesional en la carrera de Educación Especial juega un rol muy importante para la formación de los futuros docentes. Las dos modalidades de PP, virtual y presencial, convergen al momento de propiciar un acercamiento con la realidad educativa de las escuelas, identificar las necesidades y/o problemas que se presentan en cada una de ellas, así como también al reconocer las buenas prácticas educativas inclusivas. Otro punto de encuentro de estas modalidades se da en los procesos de acompañamiento de la tutora o tutor académico a los practicantes, ya que es quien gestiona la práctica de cada pareja pedagógica practicante.

Por otro lado, existen divergencias tales como: en la modalidad virtual el contacto directo entre practicantes y estudiantes es escaso, el proceso de acompañamiento por parte de la tutora o tutor profesional se limita a la coordinación de actividades y asignación de tareas para la elaboración de material didáctico, no se ejecutan los espacios de reflexión de la práctica ya que no existe el trabajo de la pareja pedagógica académica (Cátedra Integradora y Aproximación/Exploración Diagnóstica).

Todo lo señalado recalca la necesidad de diseñar una propuesta de mejora con orientaciones que respondan a las realidades de las instituciones educativas y a las necesidades de formación de futuros docentes en las prácticas preprofesionales. El documento consiste en una guía que incluye actividades sugeridas relacionadas con los resultados de aprendizaje, los mismos que deben estar articulados con el núcleo problémico y el eje integrador de cada ciclo.

Esta propuesta será implementada en el ciclo académico IS-2021 (abril-agosto 2021), ya que entrará en vigor la malla curricular rediseñada de la carrera, en la que propone que las prácticas preprofesionales inicien a partir del quinto ciclo. No obstante, considerando la naturaleza de la carrera y la importancia de generar espacios de acercamiento con las realidades educativas, el alumnado de los cuatro primeros ciclos tendrá contacto con escuelas regulares, estas actividades serán contempladas en el componente práctico-experimental de la asignatura Aproximación Diagnóstica.

Las orientaciones del documento permiten identificar casos, situaciones y/o problemáticas presentes en los contextos educativos en los que se realizarán las prácticas. Cada tutora o tutor académico organizará actividades preparatorias, entre ellas, la revisión documentada de la institución educativa y otros documentos normativos que permitirán al 
estudiantado contextualizar el marco normativo de la educación ecuatoriana antes de tener contacto con la escuela; más aún, se propiciará un análisis crítico de los distintos programas de intervención de estudiantes que se encontrarán a su cargo.

Posteriormente, el estudiantado establecerá contacto con sus tutores profesionales con quienes desarrollarán actividades enmarcadas en los ejes vertebradores de la práctica preprofesional: ayudar, acompañar y experimentar, en correspondencia con el núcleo problémico y el eje integrador de cada ciclo, para diseñar propuestas innovadoras de tipo metodológico y organizativo que serán presentadas en las escuelas de prácticas.

En el desarrollo de este estudio se presentaron algunas limitaciones, entre las más relevantes podemos mencionar la restringida información proporcionada por estudiantes y tutores profesionales, ya que al aplicar un cuestionario a través de una plataforma digital se pierde la riqueza del diálogo, la reflexión y el debate que puede abrirse a partir de un contacto cara a cara. En este documento se establece una descripción de la información obtenida en los momentos de la práctica preprofesional, en una segunda parte se analizaría el cumplimiento de los ejes vertebradores y los resultados de aprendizaje alcanzados en el alumnado de la carrera.

Es importante mencionar que este estudio podría servir como referente de diseño en evaluaciones de prácticas preprofesionales de otras instituciones; incluso, el recorrido que se hace sobre el Modelo Pedagógico y el Modelo de Prácticas Preprofesionales de la UNAE podría interesar a otras instituciones, de manera especial, cuando se hace referencia a la reflexión de la práctica y sus formas de propiciarla.

\section{Referencias}

Abreu, José Luis. (2020). Tiempos de Coronavirus: La educación en línea como respuesta a la crisis. Daena: International Journal of Good Conscience, 15(1), 1-15. Recuperado de http://www.spentamexico.org/v15-n1/A1.15(1)1-15.pdf

Arce, Débora. y Guiller, Charis. (2015). Los entornos virtuales: nuevos escenarios de enseñanza y aprendizaje en la Universidad. Actas de periodismo y comunicación, 1(1). Recuperado https://perio.unlp.edu.ar/ojs/index.php/actas/article/view/2949

Bardin, Laurence. (2002). Análisis de contenido. Madrid: Akal.

Bustos, Alfonso. y Coll, César. (2010). Los entornos virtuales como espacios de enseñanza y aprendizaje. Revista Mexicana de Investigación Educativa, 15(44), 163-184. Recuperado de https://www.redalyc.org/pdf/140/14012513009.pdf 
Clarà, Marc. y Mauri, María Teresa. (2010). El conocimiento práctico. Cuatro conceptualizaciones constructivistas de las relaciones entre conocimiento teórico y práctica educativa. Journal for the Study of Education and Development, 33(2), 131-141. Recuperado de https://dialnet.unirioja.es/servlet/articulo?codigo=3202470

Expósito, Jorge. (2020). Nuevos escenarios para la docencia universitaria. Crisis, oportunidad y algunas ideas más. Aula Magna 2.0. Recuperado de https://cuedespyd.hypotheses.org/8462

García Martínez, Andrés., Guerrero Proenza, Rey. y Romero Granados, Jesús. (2015). Buenas prácticas en los entornos virtuales de enseñanza-aprendizaje. Revista Cubana de Educación Superior, 34(3), 76-88. Recuperado de http://scielo.sld.cu/scielo.php?script=sci abstract\&pid=S0257-43142015000300006

Latorre, María José. (2006). La formación práctica universitaria del estudiante de profesorado. Enseñanza \& Teaching: Revista Interuniversitaria de Didáctica, 24, 233-253. Recuperado de http://e-spacio.uned.es/fez/eserv/bibliuned:20174/formacion practica.pdf

Lopez, Fernando. (2007). Metodología participativa en la enseñanza universitaria (2. ${ }^{\text {a }}$ ed.). Sevilla: Narcea.

Martínez, Vicente. (2017). Educación presencial versus educación a distancia. La Cuestión Universitaria, 9(9), 108-116. Recuperado de http://webcast.berkeley.edu

Pérez, María. (2008). Competencias adquiridas por los futuros docentes desde la formación inicial. Revista de Educación, (347), 347-343. Recuperado de https://dialnet.unirioja.es/servlet/articulo?codigo=2700129

Pérez Gómez, Ángel Ignacio., Soto Gómez, Encarnación. y Servan Núñez, José. (2015). Lesson Studies: re-pensar y re-crear el conocimiento práctico en cooperación. Revista Interuniversitaria de Formación del Profesorado, 29(3), 81-101. Recuperado de https://www.redalyc.org/pdf/274/27443871006.pdf

Portilla, Gladys., Abril, Hugo., Choin, David., Fraga, Odalys., Molerio, Liliana., Padilla, Javier., Pantoja, Teresa., Torres, Lucía. y Ullauri, Jaime. (2017). Modelo de práctica preprofesional de la UNAE. Cañar, Ecuador: Ed. UNAE.

Suáres Álvarez, Rebeca., Vázquez Barrio, Tamara. y Torrecillas Lacave, Teresa. (2020). Metodología y formación docente cuestiones claves para la integración de las TIC en la educación. Ambitos. Revista Internacional de Comunicación, 49, 197-216. doi: https://dx.doi.org/10.12795/Ambitos.2020.i49.12

Terranova Ruiz, Jackeline., Villafuerte Holguín, Jhonny. y Shettini Velásquez, Teresa. (2019). Sistema de prácticas preprofesionales e integración de las funciones sustantivas en las carreras de educación. Opuntia Brava, 11(2), 312-322. doi: https://doi.org/10.35195/ob.v11i2.764

Zabalza Beraza, Miguel A. (2017). El Practicum y las prácticas externas en la formación universitaria. Revista Practicum, $1(1)$. https://doi.org/10.24310/RevPracticumrep.v1i1.8254 


\section{Revista indizada en}
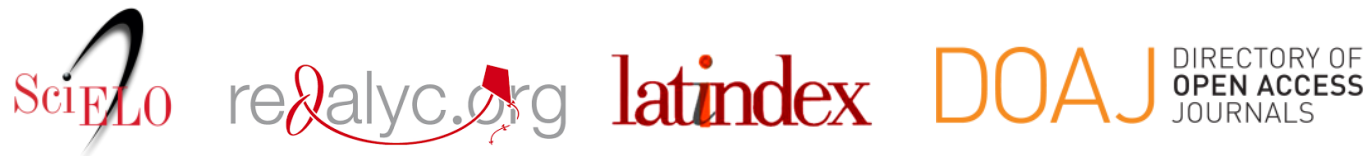

Distribuida en las bases de datos:
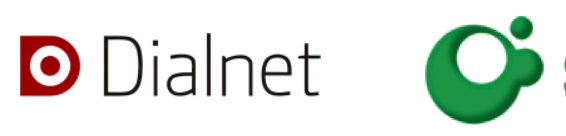
SHERPA/RøMEO

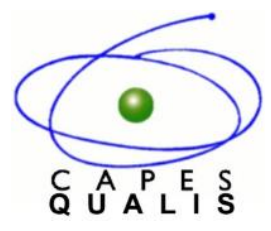

MIAR 